\title{
Importance of dynamic contrast enhanced magnetic resonance imaging for targeting biopsy and salvage treatments after prostate cancer
} recurrence

\author{
Josh Mason, PhD', Ese Adiotomre, MD', Peter Bownes, MSc', Brendan Carey, MD', Ann Henry, MD'.2 \\ 'Department of Medical Physics, Leeds Cancer Centre, St. James's University Hospital, Leeds, ${ }^{2}$ Academic Unit of Medical Physics, University of \\ Leeds, United Kingdom
}

\begin{abstract}
Purpose: Evaluate T2 weighted MRI (T2W), diffusion weighted imaging (DWI), and dynamic contrast enhanced MRI (DCE-MRI) for determining areas of prostate cancer recurrence to target biopsy or salvage treatment in patients previously treated with I-125 seed brachytherapy.

Material and methods: MRI data from 15 patients, whose primary treatment was I-125 seed brachytherapy and who were subsequently treated with partial gland salvage high-dose-rate brachytherapy were retrospectively analyzed. Two radiologists independently reviewed imaging on two occasions blinded to clinical and biopsy information. At first review, the T2W and DWI sequences were assessed for likely presence of tumor and at second review, the additional DCE-MRI sequence was assessed. Results were recorded and compared on a prostate diagram divided into 12 sectors (quadrants at each of base, mid-gland, and apex) plus seminal vesicles (SV).

Results: Number of patients for whom recurrence was visible was 7/15 for T2W, 6.5/15 for DWI, and 15/15 for DCE-MRI (average of results for the two radiologists). Approximately, half of the sectors identified as showing recurrence were at the anterior base of the prostate.

Conclusions: In prostate cancer patients previously treated with I-125 permanent seed implants, DCE-MRI is superior to T2W and DWI in defining areas of recurrence, and should be used to target biopsy and for treatment planning of focal salvage therapies.

J Contemp Brachytherapy 2018; 10, 6: 570-572 DOI: https://doi.org/10.5114/jcb.2018.79667
\end{abstract}

Key words: cancer recurrence, multi-parametric MRI, prostate brachytherapy, targeted biopsy.

\section{Purpose}

Permanent seed implant brachytherapy is a standard primary treatment for low- or intermediate-risk localized prostate cancer. A proportion of patients will experience recurrence and combined with the high incidence of prostate cancer, this means that significant numbers of men with non-metastatic prostate cancer will develop biochemical failure following primary radiation treatment. Multi-parametric magnetic resonance imaging (mp-MRI) has an important role in localizing areas of recurrence to guide targeted biopsies and planning salvage treatments. The ability to perform targeted partial gland salvage re-treatments using brachytherapy as opposed to re-treating the whole prostate gland may allow a higher dose to be given to the tumor, while reducing dose to organs at risk that have already been irradiated by the primary treatment [1]. The PI-RADs v2 radiology guidelines [2] for reporting mp-MRI before therapy recommend that diffusion weighted imaging (DWI) is the primary determining sequence in the peripheral zone of the prostate and that T2 weighted MRI (T2W) is the primary determining sequence in the transition zone of the prostate, while dynamic contrast enhanced MRI (DCE-MRI) has only a minor role. However, after radiotherapy, radiation induced fibrosis may make T2W and DWI less useful. DCE-MRI demonstrates cancer induced neo-angiogenesis and may be of more value [3,4]. In addition, for patients previously treated with seed implant brachytherapy, the seeds may create artifact that further reduces the value of DWI.

This study of patients previously treated with seed implant brachytherapy compares T2W, DWI and DCEMRI in terms of their usefulness for localizing areas of prostate cancer recurrence.
Address for correspondence: Josh Mason, PhD, Department of Medical Physics, St James's University Hospital, Leeds LS9 7TF, United Kingdom, phone: +44 11320 67905, 凶 e-mail: joshua.mason@nhs.net
Received: 24.08 .2018

Accepted: 23.10 .2018

Published: 28.12.2018 


\section{Material and methods}

Fifteen patients who had been treated with I-125 seed implant monotherapy (145 Gy minimum peripheral dose) between 2002 and 2013, and were subsequently treated with partial gland salvage high-dose-rate (HDR) prostate brachytherapy between 2010 and 2018 were retrospectively assessed. These patients were classified as low- or intermediate-risk at original diagnosis. The mean time from primary treatment to salvage treatment was 8 years (range, 4-14 years). At the time of the salvage treatment, the average age was 70 (range, 63-78 years). Mean prostate-specific antigen was 11 (range, 3-20) at initial treatment and 5 (range, 1-9) at relapse. All patients were treated with a single 19 Gy fraction of focal salvage HDR brachytherapy, with the focal target determined using mp-MRI data and biopsy results. Targeted trans-rectal ultrasound template biopsy was performed after mp-MRI and consisted of systematic sampling with additional samples taken from areas suspicious for recurrence in the mp-MRI.

The mp-MRI acquisition was performed 2-6 months before salvage treatment and consisted of T2W, DWI, and DCE-MRI. Scans were performed on Avanto or Aera (Siemens AG, Munich, Germany) 1.5T scanners, using phased-array pelvic and spine coils for signal reception. T2W MRI used a turbo-spin echo (SE) sequence with repetition time (TR) $3200 \mathrm{~ms}$, echo time (TE) $87 \mathrm{~ms}$, echo train length 13, $0.6 \mathrm{~mm}$ pixel size, slice thickness $3 \mathrm{~mm}$. DWI MRI used a single shot SE-echo planar imaging (EPI) sequence with TR $3000 \mathrm{~ms}$, TE $81 \mathrm{~ms}, 1.5 \mathrm{~mm}$ pixel size, slice thickness $4 \mathrm{~mm}$, b-values $0,150,1000 \mathrm{~s} / \mathrm{mm}^{2}$. Raw DWI images as well as the apparent diffusion coefficient (ADC) maps generated by the scanner (using all three b-values) were reviewed for this study. Some data sets also had calculated $\mathrm{b}=1400 \mathrm{~s} / \mathrm{mm}^{2}$ images. DCE MRI used a 3D spoiled gradient echo sequence with TR $4.5 \mathrm{~ms}$, TE $1.6 \mathrm{~ms}$, flip angle $15^{\circ}, 1.4 \times 1.4 \times 3 \mathrm{~mm}$ pixel size, $30 \mathrm{ac}$ quisitions with 10s-time resolution, and a bolus injection of $0.1 \mathrm{mmol} / \mathrm{kg}$ Dotarem ${ }^{\circledR}$ (Guerbet Group, Villepinte, France). Raw DCE images were reviewed for areas of early enhancement.

MRI images were reviewed on two occasions by two radiologists with over 20 years and 5-year experience of prostate MRI, respectively. At the first review, the T2W and DWI sequences were assessed for likely presence of tumor and at the second review, undertaken at least
2 weeks later, the additional DCE sequence was assessed. DCE sequences were assessed blinded to the T2W/DWI sequences to reduce recall bias and as the DCE-MRI images showed sufficient anatomical detail to allow the prostate to be clearly visualized (Figure 1). Each review was performed independently by the two consultant radiologists, blinded to clinical and biopsy information. Results were recorded and compared on a prostate diagram divided into 12 sectors (quadrants at each of base, mid-gland and apex) plus seminal vesicles (SV). Each sector was scored as either positive or negative for suspicion of recurrence. After this analysis was complete, the biopsy reports for each patient were checked to ensure that sectors identified as positive or negative were consistent with the biopsy results.

\section{Results}

The first radiologist found recurrence visible in 6/15 patients for T2W, 5/15 for DWI, and 15/15 for DCE-MRI. The second radiologist found recurrence visible in 8/15 patients for T2W, 8/15 for DWI, and 15/15 for DCE-MRI. For the 3 patients in which recurrence was scored differently by the two radiologists, the area of recurrence identified was smaller for T2W/DWI compared to DCE-MRI. For two of the patients where recurrence was visible on DWI, the DWI visible recurrence was confined to the SV where there are no seeds present.

From the sector-based analysis, two patients had SV only recurrence. For the remaining 13 patients, the recurrence involved a median of $2 / 12$ prostate sectors (range, $1 / 12-5 / 12$ ) predominantly in anterior base sectors; Figure 2 shows the total number of recurrences detected in each sector. The total number of involved sectors was 34 and 44 for the first and second radiologist, respectively. Per patient, the median number of sectors scored differently was 2 (range, 0-5). These differences were subsequently reviewed, and, in all cases, the two radiologists agreed on at least one sector, indicating that the differences were down to the extent of the recurrence (whether or not to include adjacent sectors). Following the imaging review, targeted biopsy results were reviewed, and, in all cases, the areas identified as positive or negative for recurrence were consistent with the biopsy results (as this was a retrospective imaging study it was only possible to verify the recurrence location in terms of gross anatomical location rather than verify each sector individually).

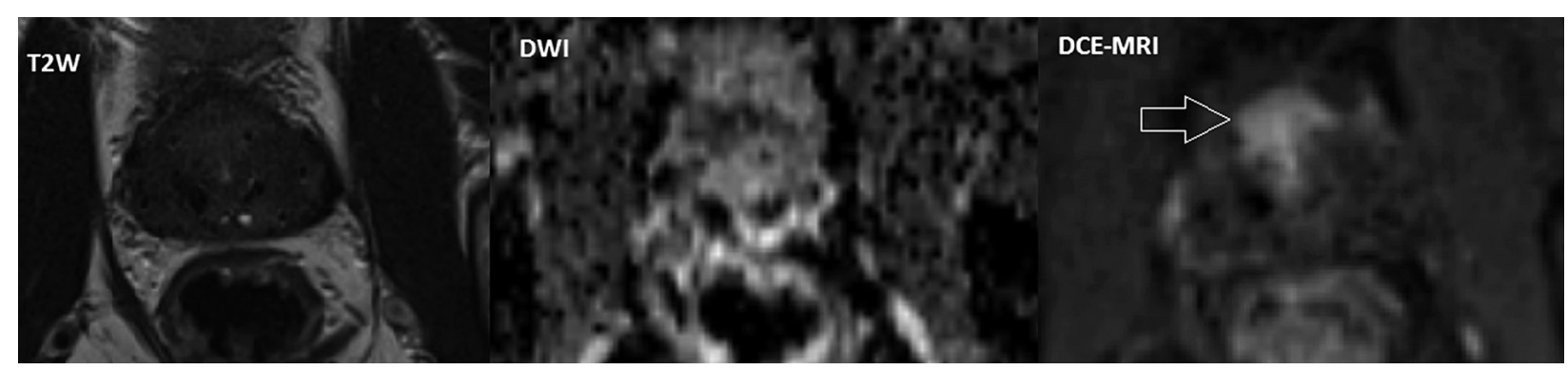

Fig. 1. Mp-MRI images for a patient whose original treatment was I-125 seeds, anterior tumor is visible as increased enhancement on DCE-MRI. In the DWI ADC map there are possible darker areas of restricted diffusion, but artifacts generated by the implanted seeds make this image hard to interpret. The tumor is not visible on the T2W images. Targeted biopsy confirmed presence of tumor in anterior cores only 

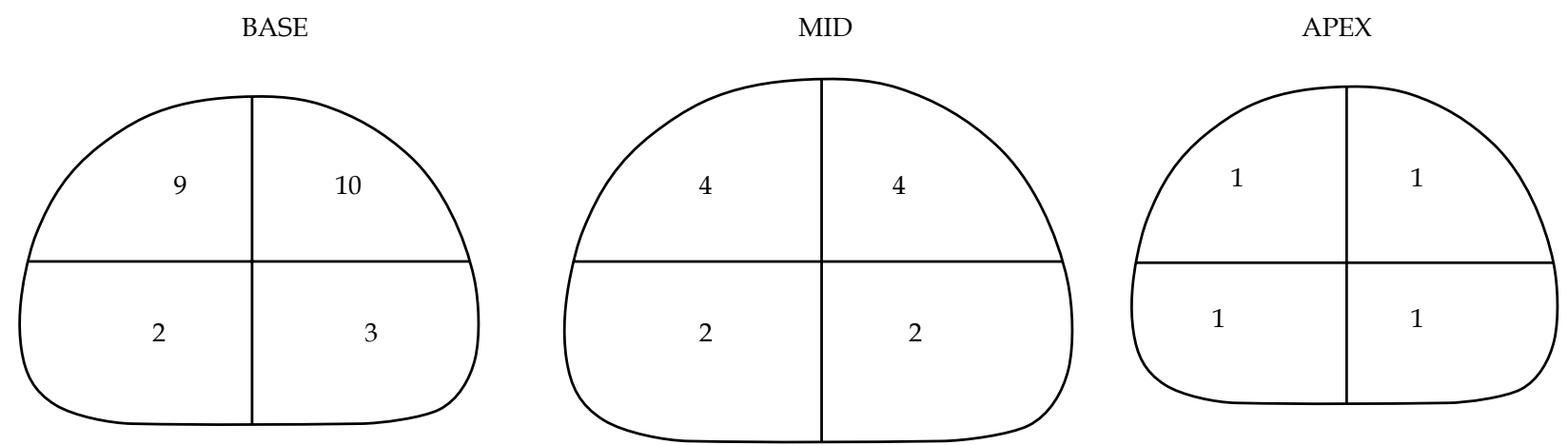

Fig. 2. The number of times each sector was identified as involved for the 15 I-125 seed patients. The numbers given are the average values for the two radiologists who reviewed the images. Four patients also had recurrence in the seminal vesicles

\section{Discussion}

This study has compared T2W, DWI, and DCE-MRI for localizing areas of prostate cancer recurrence in patients previously treated with I-125 seed implant brachytherapy. The usefulness of DCE-MRI in assessing recurrence in prostate cancer patients treated with external beam radiotherapy has been assessed before [5,6,7] but this study is the first to focus purely on patients with I-125 seed implant for their primary treatment, and specifically for the purpose of planning focal salvage brachytherapy treatments. A study by Donati et al. [7] did include some seed implant patients and concluded that DCE-MRI was helpful in these cases, although not adding value for patients treated with external beam radiotherapy. That study also focused primarily on imaging for detecting recurrence rather than for planning treatments. Mp-MRI has a crucial role both in guiding targeted biopsy and in defining the area for focal treatment, allowing dose escalation to the area of recurrence, whilst minimizing dose to organs at risk.

In this study, DCE-MRI could be used to visualize the area of recurrence in all 15 patients, whereas $\mathrm{T} 2 \mathrm{~W}$ and DWI were only useful in approximately half of patients. The poorer performance of DWI in seed implant patients is due to artifact generated by the seeds in addition to the effects of the original treatment. Two radiologists reviewed images for this study and their results were reasonably consistent, although differences in their evaluation of the extent of the tumor could translate to differences in volumes targeted for focal salvage treatments. This points to a limitation of DCE-MRI in that regions of enhancement are not generally very clearly defined. A further limitation of this study is that DCE-MRI can show abnormal enhancement due to benign prostatic hyperplasia or inflammation, and this could have caused some of the areas of recurrence to be overestimated. However, in planning focal salvage treatments it is preferable to treat all areas suspicious for recurrence. Recurrences were much more commonly seen at the anterior base and this is consistent with studies that show this area of the prostate tends to receive lower dose $[8,9]$.

\section{Conclusions}

In prostate cancer patients previously treated with I-125 permanent seed implants, DCE-MRI is superior to
T2W and DWI in defining areas of recurrence, and should be used to target biopsy and for treatment planning of focal salvage therapies.

\section{Disclosure}

Authors have received industry funding from BXTAccelyon and Elekta to travel and participate in educational meetings.

\section{References}

1. Duijzentkunst DS, Peters M, van Zyp JvdV et al. Focal salvage therapy for local prostate cancer recurrences after primary radiotherapy: a comprehensive review. World J Urol 2016; 34: 1521-1531.

2. Weinreb JC, Barentsz JO, Choyke PL et al. PI-RADS prostate imaging-reporting and data system: 2015, version 2. Eur Urol 2016; 69: 16-40.

3. Wu L, Xu J-R, Gu H et al. Role of magnetic resonance imaging in the detection of local prostate cancer recurrence after external beam radiotherapy and radical prostatectomy. Clin Oncol (R Coll Radiol) 2013; 25: 252-264.

4. Roy C, Foudi F, Charton J et al. Comparative sensitivities of functional MRI sequences in detection of local recurrence of prostate carcinoma after radical prostatectomy or external-beam radiotherapy. AJR Am J Roentgenol 2013; 200: W361-W368.

5. Haider MA, Chung P, Sweet J et al. Dynamic contrast-enhanced magnetic resonance imaging for localization of recurrent prostate cancer after external beam radiotherapy. Int J Radiat Oncol Biol Phys 2008; 70: 425-430.

6. Rouvière $\mathrm{O}$, Valette $\mathrm{O}$, Grivolat $\mathrm{S}$ et al. Recurrent prostate cancer after external beam radiotherapy: value of contrast-enhanced dynamic MRI in localizing intraprostatic tumor - correlation with biopsy findings. Urology 2004; 63: 922-927.

7. Donati O, Jung S, Vargas $\mathrm{H}$ et al. Multiparametric Prostate MR imaging with T2-weighted, diffusion-weighted, and dynamic contrast-enhanced sequences: are all pulse sequences necessary to detect locally recurrent prostate cancer after radiation therapy? Radiology 2013; 268: 440-450.

8. Nasser NJ, Wang Y, Borg J, Saibishkumar EP. Sector analysis of dosimetry of prostate cancer patients treated with lowdose-rate brachytherapy. Brachytherapy 2014; 13: 369-374.

9. Mohamed Yoosuf AB, Workman G, O'Toole MM et al. Sector analysis of 125I permanent prostate brachytherapy provides a rapid and effective method of evaluating and comparing preand post-implant dosimetry. Brachytherapy 2013; 12: 254-259. 